\title{
Transcriptomics analysis of sirolimus treatment in lupus nephritis
}

\author{
DONGDONG WANG ${ }^{1 *}, \mathrm{XIAO} \mathrm{CHEN}^{2^{*}}, \mathrm{MENG} \mathrm{FU}^{1}$ and ZHIPING LI ${ }^{1}$ \\ ${ }^{1}$ Department of Pharmacy, Children's Hospital of Fudan University, Shanghai 201102; ${ }^{2}$ Department of Pharmacy, \\ The People's Hospital of Jiangyin, Jiangyin, Jiangsu 214400, P.R. China
}

Received December 17, 2018; Accepted April 8, 2019

DOI: $10.3892 / \mathrm{mmr} .2019 .10238$

\begin{abstract}
Lupus nephritis (LN) is one of the principal causes of mortality and disability in patients with systemic lupus erythematosus. Sirolimus has been used to treat patients with LN; however, the effects and mechanism of sirolimus in these patients remains unclear. The present study aimed to elucidate the therapeutic effects and mechanisms of sirolimus in LN mice using low, medium and high doses of sirolimus (0.1, 0.3 and $1 \mathrm{mg} / \mathrm{kg}$, respectively). The survival probability and kidney index were calculated, and renal fibrosis was determined using Masson's Trichrome staining. The expression levels of E-cadherin, $\alpha$-smooth muscle actin ( $\alpha$-SMA) and vimentin were assessed via immunofluorescence analysis. Transcriptome analysis of control and sirolimus-treated LN mice was performed using RNA-sequencing, differentially expressed gene (DEG) identification and annotation, and Gene Ontology (GO) functional and Kyoto Encyclopedia of Genes and Genomes (KEGG) pathway enrichment. The results suggested that a medium dose of sirolimus alleviated renal fibrosis and increased the survival rates of mice with $\mathrm{LN}$ $(\mathrm{P}<0.05)$. Furthermore, transcriptome analysis revealed 334 DEGs associated with LN, 176 of which were upregulated and 158 were downregulated. Following GO functional enrichment, 'biological process', 'molecular function' and 'cellular component' terms were identified. A total of $10 \mathrm{KEGG}$ pathways were enriched, with 'cytokine-cytokine receptor interaction' and 'interleukin-17 signaling pathway' being significantly enriched $(\mathrm{P}<0.05)$. To the best of our knowledge, the present study is the first to conduct transcriptome analysis of LN mice treated with sirolimus, and demonstrated that a dose of $0.3 \mathrm{mg} / \mathrm{kg}$ exerted the greatest therapeutic effects.
\end{abstract}

Correspondence to: Professor Zhiping Li, Department of Pharmacy, Children's Hospital of Fudan University, 399 Wanyuan Road, Shanghai 201102, P.R. China

E-mail: zplifudan@126.com

*Contributed equally

Key words: transcriptomics, analysis, sirolimus, treatment, lupus nephritis

\section{Introduction}

Systemic lupus erythematosus (SLE) is a chronic autoimmune disease characterized by the production of large quantities of autoantibodies. These antibodies are deposited in the vascular bed of target tissues and organs, including the glomeruli and the renal microvasculature, leading to systemic inflammation and lupus nephritis (LN) (1-6). LN is the principal cause of morbidity and mortality in patients with SLE (6), and as of resistance to existing medications, the development of novel treatments is required (7).

Sirolimus (also known as rapamycin) is an inhibitor of the mammalian target of rapamycin (mTOR) $(8,9)$, and has been widely used in transplantation patients to prevention allograft rejection $(10,11)$. It has also been reported as an effective treatment for pediatric tuberous sclerosis complex $(12,13)$, cirrhosis or hepatocellular carcinoma accompanied with psoriasis following liver transplantation (14), and tumor recurrence in patients with hepatocellular carcinoma (15).

Transcriptomics analysis has been used to study the therapeutic mechanisms of LN (16). Recently, sirolimus has been administered as a treatment for patients with LN (17); however, its effects on patients with $\mathrm{LN}$, in addition to its mechanism of action, are yet to be clarified. In the present study, the regulatory mechanism of sirolimus in LN was elucidated using transcriptomics analysis, which was performed using RNA-sequencing, differentially expressed gene (DEG) identification and annotation, Gene Ontology (GO) functional and Kyoto Encyclopedia of Genes and Genomes (KEGG) pathway enrichment.

\section{Materials and methods}

Animal experiments. Murphy Roths Large/lymphoproliferation strain (MRL/lpr) mice an established model of LN, were selected as the animal model for the present study. Specific pathogen free (SPF) grade female MRL/lpr mice ( $\mathrm{n}=28$ ) weighing 16-20 $\mathrm{g}$ at 12 weeks of age, were obtained from Shanghai SLAC Laboratory Animal Co., Ltd. (Shanghai, China). Age and weight matched SPF female C57BL/6 mice ( $\mathrm{n}=7$; Shanghai SLAC Laboratory Animal Co., Ltd.) were used as the normal control group (NC). MRL/lpr mice were randomly divided into the $\mathrm{LN}$ control group ( $\mathrm{LN}, \mathrm{n}=7$ ), sirolimus low-dose treatment group (SIRL, $\mathrm{n}=7$ ), sirolimus medium-dose treatment group (SIRM, $\mathrm{n}=7$ ) and the sirolimus high-dose treatment group (SIRH, $\mathrm{n}=7$ ), respectively. Mice of the low, medium and high-dose treatment 
groups were administered sirolimus (Shanghai Topbiochem Technology Co., Ltd.) at a dose of $0.1,0.3$ and $1 \mathrm{mg} / \mathrm{kg}$ per day, respectively, by intragastric administration for 4 weeks. Control groups, including the $\mathrm{NC}$ and $\mathrm{LN}$ groups, received daily intragastric administration of equal amounts of $1 \%$ sodium carboxymethylcellulose for 4 weeks. The present study was approved by the Animal Care and Use Committee of the Children's Hospital of Fudan University (Shanghai, China), and complied with our institutional regulations. The survival rate in each treatment group was calculated by dividing the number of mice that survived until the end of the experiments by the amount of animals at the start.

Sample collection. Mice were anesthetized with chloral hydrate $(400 \mathrm{mg} / \mathrm{kg})$ by intraperitoneal injection. Subsequently, the mice were sacrificed by cervical dislocation and their kidneys were removed and weighed. The kidney index, which represented the relative kidney size, was the ratio between the average kidney weight and body weight. The fresh kidneys were fixed in $4 \%$ paraformaldehyde at room temperature for $24 \mathrm{~h}$, and the residual kidney tissues were snap frozen in liquid nitrogen, and stored at $-80^{\circ} \mathrm{C}$ until use.

Masson trichome staining. Following fixation with paraformaldehyde, the samples were embedded in paraffin and cut into 4- $\mu$ m-thick sections. Then, the sections were deparaffinized in xylene at room temperature for $20 \mathrm{~min}$, rehydrated in a descending ethanol series (100 and 75\% ethanol at room temperature for $5 \mathrm{~min}$ ) and washed in water prior to overnight incubation in potassium dichromate at room temperature. After washing in water, the sections were stained with hematoxylin iron solution for $3 \mathrm{~min}$ at room temperature, followed by $0.5 \%$ acid alcohol, and washed in water once more. Samples were stained with Ponceau S solution for $5 \mathrm{~min}$ at room temperature, and subsequently rinsed in water. Finally, samples were stained using phosphomolybdic acid for $1 \mathrm{~min}$, followed by aniline blue solution for $3 \mathrm{~min}$, and washed with $1 \%$ acetic acid solution at room temperature. The sections were dehydrated using $100 \%$ alcohol, vitrified in xylene, and mounted with neutral gum. The accumulation of collagen fibers (blue) was determined using Image-Pro Plus 6.0 image analysis software (Media Cybernetics, Inc., Rockville, MD, USA).

Immunofluorescence. The $4 \mu \mathrm{m}$ sections were deparaffinized in xylene, rehydrated using a descending ethanol series $(100,85$ and $75 \%$ ethanol, respectively) and washed in water. Following antigen retrieval, the spontaneous fluorescence quencher (Wuhan Servicebio Technology Co., Ltd; cat. no. G1221) was added for $5 \mathrm{~min}$ at room temperature, and the samples were rinsed with water for $10 \mathrm{~min}$. The sections were subsequently blocked using bovine serum albumin (Wuhan Servicebio Technology Co., Ltd.) for $30 \mathrm{~min}$ at room temperature, and incubated with antibodies against E-cadherin (1:100; Wuhan Servicebio Technology Co., Ltd.; cat. no. GB12082), $\alpha$-smooth muscle actin ( $\alpha$-SMA, 1:500; Wuhan Servicebio Technology Co., Ltd.; cat. no. GB13044) and vimentin (1:200, Wuhan Servicebio Technology Co., Ltd.; cat. no. GB11192) at $4^{\circ} \mathrm{C}$ overnight. Following the primary incubation, the sections were washed and incubated with the corresponding conjugated secondary antibodies with Cy3 (1:300, Wuhan
Servicebio Technology Co., Ltd.; cat. no. GB21303) and Alexa Fluor 488 (1:400, Wuhan Servicebio Technology Co., Ltd.; cat. no. GB25301) at room temperature, in the dark room for $50 \mathrm{~min}$. The sections were stained with DAPI solution and incubated in the dark at room temperature for $10 \mathrm{~min}$, prior to quenching using an anti-fluorescence quenching tablet. Images were acquired using a fluorescence microscope (Nikon Eclipse $\mathrm{C} 1$; Nikon Corporation).

RNA library construction and sequencing. Our preliminary analysis using various doses of sirolimus revealed that medium-dose sirolimus treatment had a therapeutic effect on LN. Therefore, the kidneys of mice in the LN and SIRM groups were used to conduct transcriptome analysis. RNA library construction and sequencing were performed in triplicate by Suzhou Base Pair Biotechnology (Suzhou, China) using an Illumina HiSeq $\mathrm{X}^{\mathrm{TM}}$ Ten Sequencing System (Illumina, Inc., San Diego, CA, USA).

DEG identification and annotation. Raw reads were generated from image data and stored in FASTQ format. Raw data were filtered to remove adaptor-contaminated and low quality sequences, and to obtain clean reads (18). The quality of the clean reads was checked using FastQC (version 0.11.4; https://www.bioinformatics.babraham. ac.uk/projects/fastqc/) and aligned to the reference genome Ensemble-GRCM38 using Hisat2 (version 2.1.0) (19); $\leq 2$ base mismatches or read gaps were permitted in the alignment. Gene coverage was calculated as the percentage of genes covered by reads, and gene functional annotation was performed using the ANNOVAR tool (20). DEGs were identified using DEGseq panormalage (21) based on a negative binomial distribution. Gene expression levels were measured based on fragments per kilobase of transcript per million read pairs and count values. The SIRM group reflected the 'case' and the LN group was the 'control' for analysis. The results represented the case gene expression relative to that of the control. Genes with an adjusted $\mid \log 2$ (fold change) $\mid>1$ and $\mathrm{P}<0.05$ were identified as DEGs. KEGG pathway and GO annotation of DEGs was performed using Kobas with the KEGG (22), and GO (23) databases, respectively. Significantly enriched KEGG pathways and GO terms were identified by $\mathrm{P}<0.05$.

\section{Results}

Sirolimus treatment in mice with LN. As demonstrated in Fig. 1A, the SIRM group revealed a high probability of survival, while animals of the SIRH group had succumbed. As presented in Fig. 1B, the LN group exhibited a markedly higher kidney index compared with the NC group; data of the SIRH group could not be obtained were thus emitted from subsequent analysis. Of note, the SIRM group possessed a significantly lower kidney index compared with the LN group. Compared with the NC group, fibrosis was significantly promoted in the LN group, as demonstrated by Masson Trichome staining. Compared with the LN group, significantly reduced renal fibrosis was observed in the SIRM group (Fig. 1C). Therefore, medium-dose sirolimus treatment was proposed to possess notable therapeutic potential against $\mathrm{LN}$. 
A

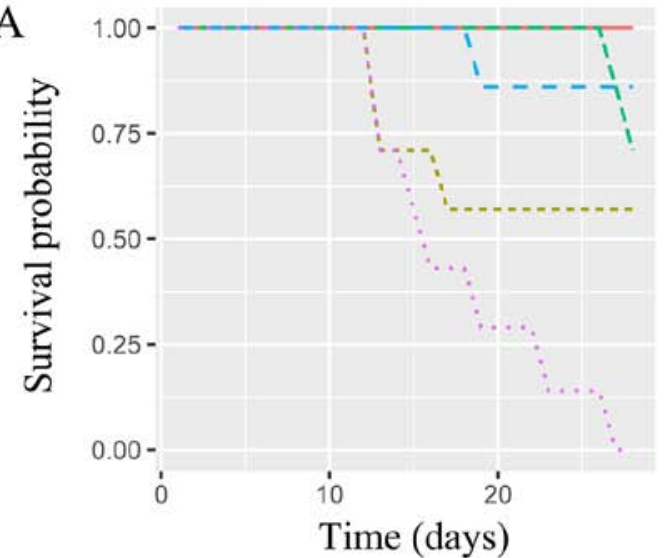

C

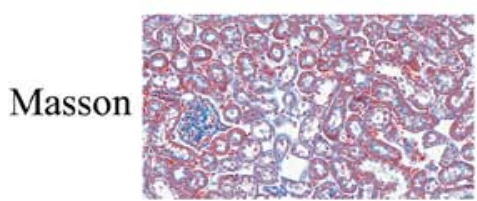

$\mathrm{NC}$

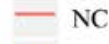

$\cdots \mathrm{LN}$

-- SIRL

$--\operatorname{SIRM}$

$\cdots$ SIRH

Time (days)

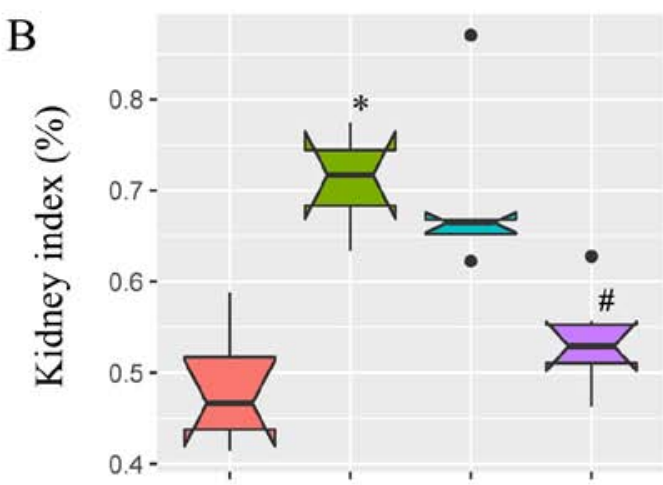

NC LN SIRL SIRM

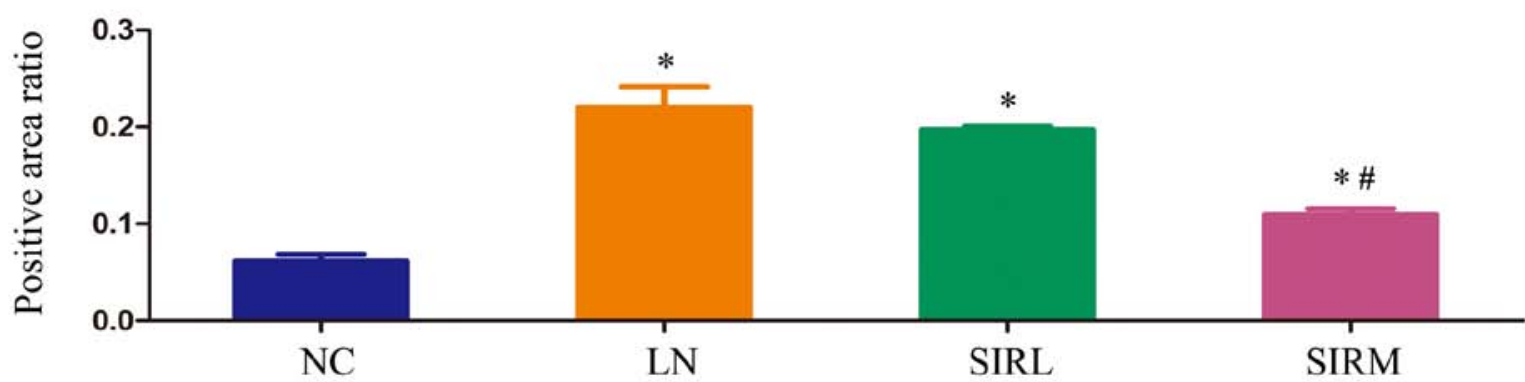

Figure 1. Sirolimus treatment in mice with LN. (A) Survival probability; (B) kidney index, (kidney index=average kidney weight/body weight) and (C) Masson's Trichome staining ( $\mathrm{n}=3$ ). Scale bar, $100 \mu \mathrm{m}$. " $\mathrm{P}<0.05$ vs. $\mathrm{NC}$ group; ${ }^{\text {}} \mathrm{P}<0.05$ vs. $\mathrm{LN}$ group. NC, normal control; $\mathrm{LN}$, lupus nephritis control group; SIRL, sirolimus low-dose treatment group; SIRM, sirolimus medium-dose treatment group; SIRH, sirolimus high-dose treatment group.

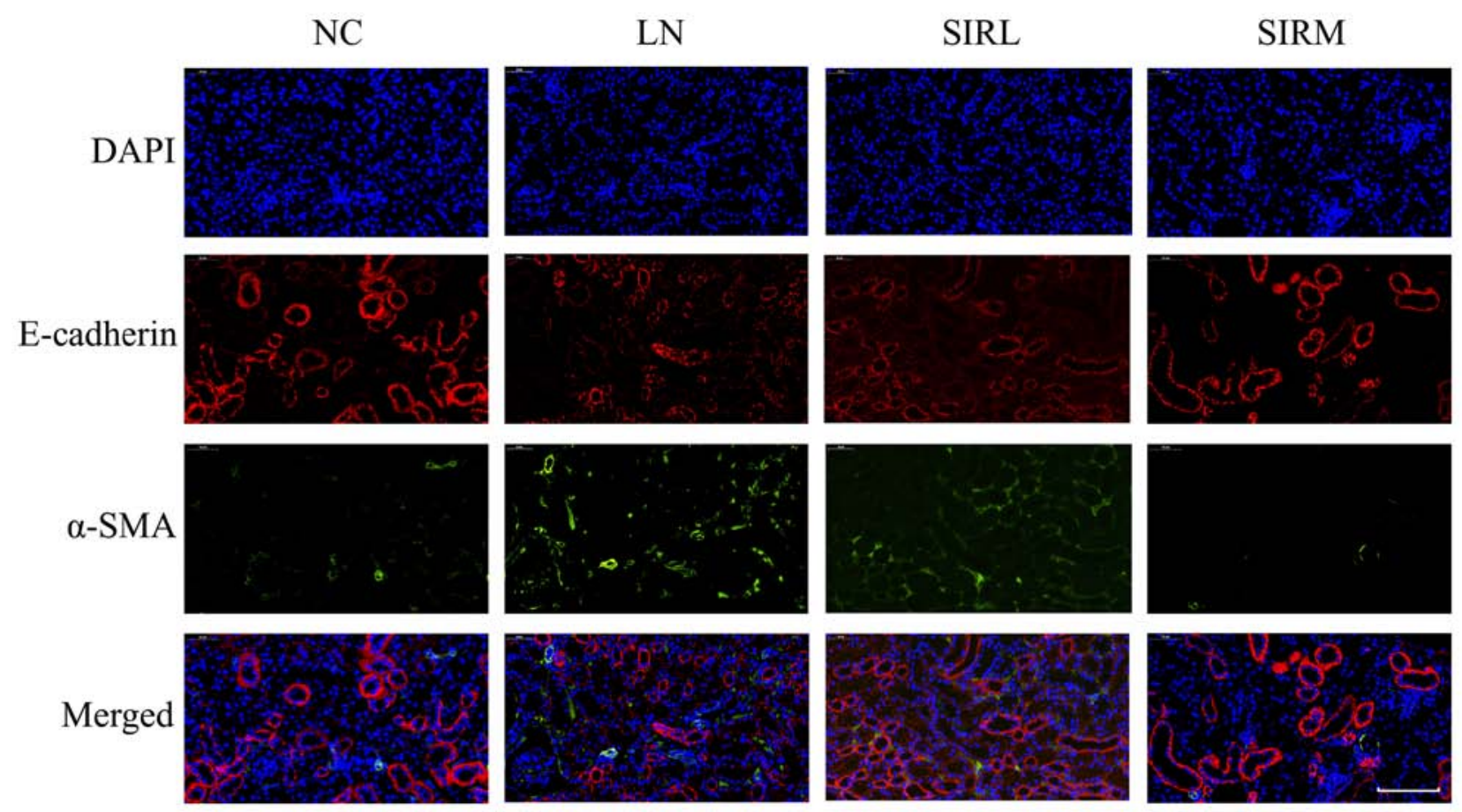

Figure 2. Effects of sirolimus on the expression levels of E-cadherin and $\alpha$-SMA in lupus nephritis. Scale bar, $100 \mu \mathrm{m}$. NC, normal control; LN, lupus nephritis control group; SIRL, sirolimus low-dose treatment group; SIRM, sirolimus medium-dose treatment group; $\alpha$-SMA, $\alpha$-smooth muscle actin. 


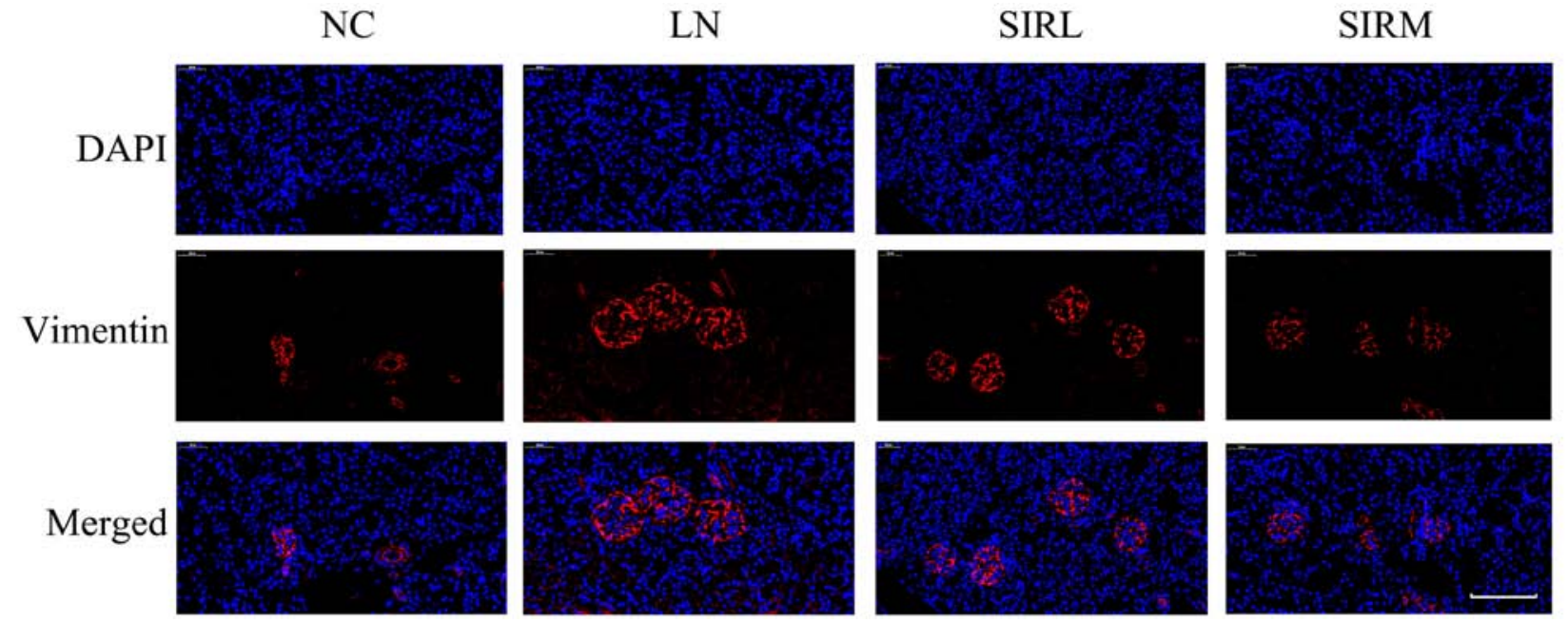

Figure 3. Effects of sirolimus on vimentin expression in lupus nephritis. Scale bar, $100 \mu \mathrm{m}$. NC, normal control; LN, lupus nephritis control group; SIRL, sirolimus low-dose treatment group; SIRM, sirolimus medium-dose treatment group.

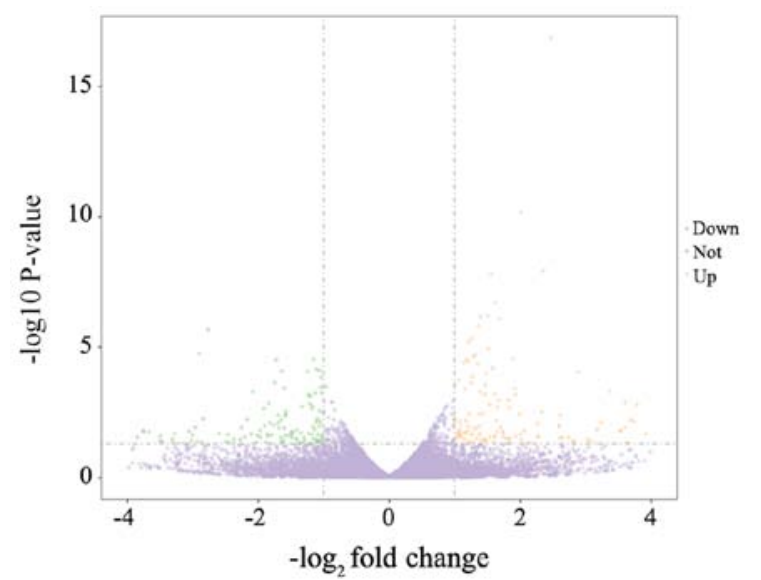

Figure 4. Differentially expressed genes of the SIRM and LN groups identified by $\mid \log 2$ (fold change) $\mid>1$ and adjusted to $\mathrm{P}<0.05$. LN, lupus nephritis control group; SIRM, sirolimus medium-dose treatment group.

As presented in Fig. 2, the LN group possessed lower E-cadherin expression levels and increased $\alpha$-SMA expression levels compared with the NC group; the SIRM group exhibited upregulated E-cadherin expression, and reduced $\alpha$-SMA expression when compared with the LN and low-dose groups. The LN group exhibited increased vimentin expression compared with NC group, however, downregulated vimentin expression was reported compared with the LN and low-dose groups (Fig. 3). These results are consistent with the findings presented in Fig. 1, suggesting that medium-dose treatment was more effective, and that the results could be used for future analysis of the transcriptome.

Transcriptomics analysis of sirolimus treatment in LN. The results of the present study represent gene expression in the SIRM group relative to that in the LN group. Transcriptomics analysis indicated a total of 334 DEGs, of which there were 176 upregulated and 158 downregulated genes (Fig. 4). Upon GO functional enrichment, 'biological process', 'molecular function' and 'cellular component' terms were identified $(\mathrm{P}<0.05)$ and can be found in Fig. 5. A total of $10 \mathrm{KEGG}$ pathways were enriched, of which 'cytokine-cytokine receptor interaction' and 'interleukin (IL)-17 signaling pathway' were significantly enriched $(\mathrm{P}<0.05$; Fig. 6$)$.

\section{Discussion}

SLE is a systemic disease that can affect several organs. During the course of the disease, there is a high incidence of kidney damage, which is referred to as LN. Kidney damage is a considerable cause of mortality and disability in patients with SLE, and includes glomerular, tubular, renal interstitial and blood vessel destruction $(5,24)$.

Expansion and/or disruption of the intraglomerular extracellular matrix (ECM) is a recognized phenomenon which occurs during the development of $\mathrm{LN}$, and may influence the deposition of immune complexes in the renal system. The factors mediating this process, and the structure and composition of the affected regions require further investigation. Increased or altered synthesis of ECM components and/or their accumulation could potentially serve a role in $\mathrm{LN}$, although the contribution made by each of these factors remains unknown (25).

ECM accumulation results in mesangial expansion, tubulointerstitial fibrosis and irreversible deterioration of renal function (26-29). The epithelial-mesenchymal transition (EMT) of renal tubular epithelial cells is one of the underlying mechanisms of renal fibrosis, and encompasses a range of events whereby epithelial cells no longer exhibit certain epithelial traits, including E-cadherin expression. Instead, the cells acquire characteristics typical of mesenchymal cells, including the expression of $\alpha$-SMA $(30,31)$. In addition, vimentin has also been reported as a potential novel biomarker of renal fibrosis (32).

In the present study, the SIRM group exhibited a low kidney index, reduced fibrosis, downregulated expression of EMT-associated factors and vimentin, and improved survival in LN mice. To reveal the molecular mechanism underlying the effects of sirolimus in LN mice, transcriptomic analysis of sirolimus-treated mice was conducted using RNA-sequencing and DEG identification and annotation. Upon GO functional 


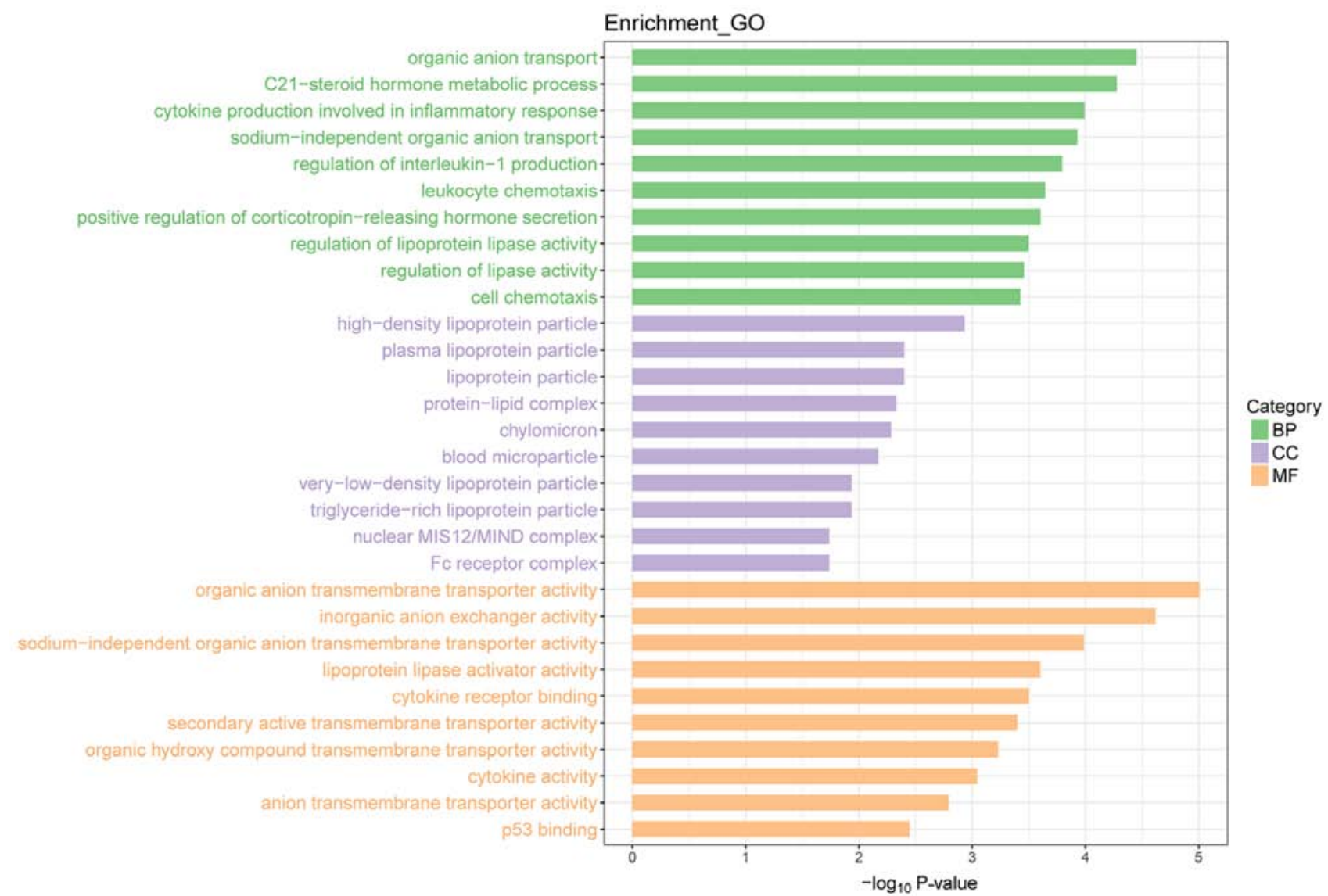

Figure 5. Enriched GO terms of differentially expressed genes. BP, biological process; CC, cellular component; GO, Gene Ontology; MF, molecular function.

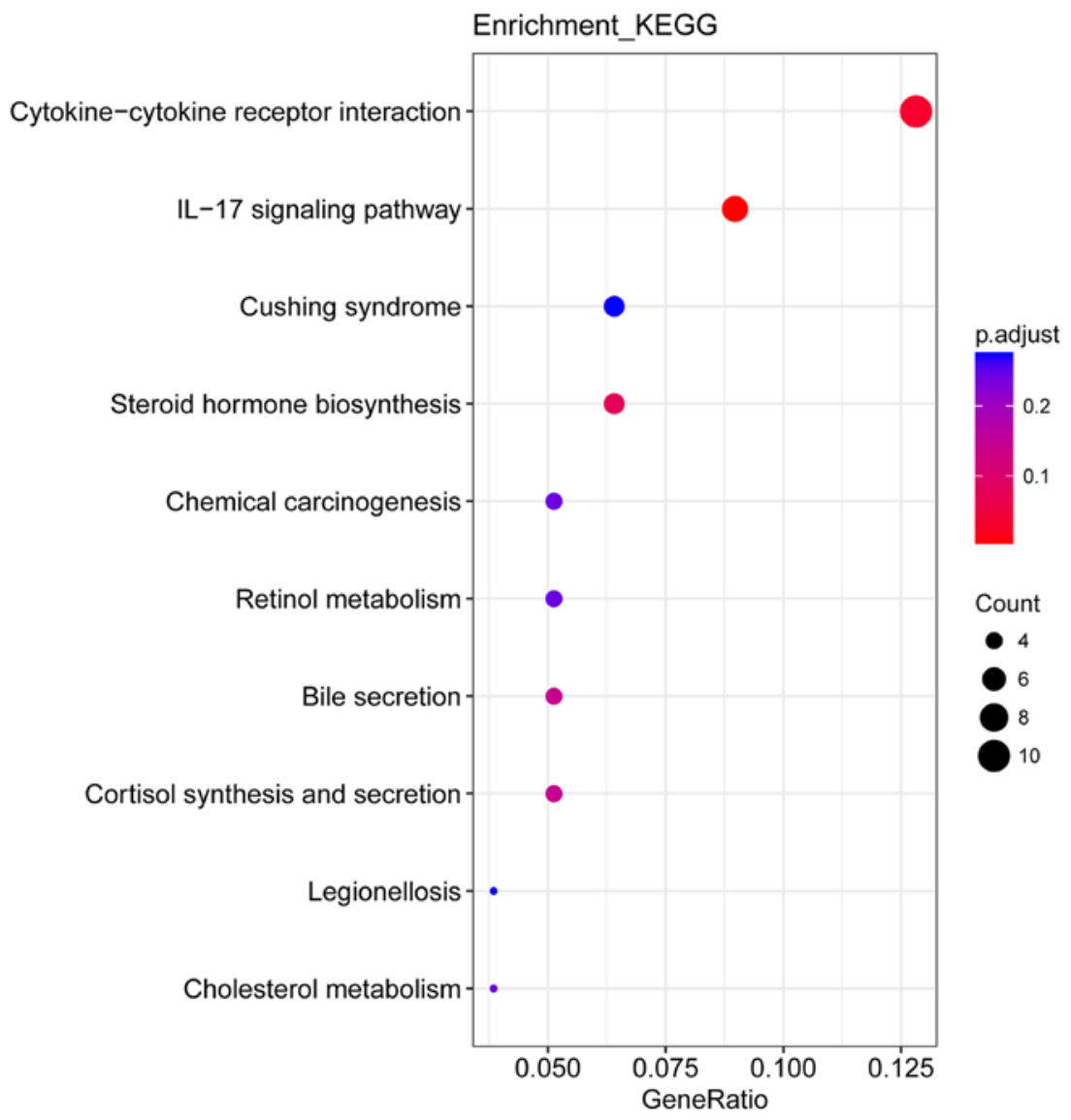

Figure 6. KEGG pathway enrichment of differentially expressed genes. IL-17, interleukin-17; KEGG, Kyoto Encyclopedia of Genes and Genomes. 
enrichment, terms associated with 'biological process', 'molecular function' and 'cellular component' were identified. A total of $10 \mathrm{KEGG}$ pathways were enriched, including 'Cytokine-cytokine receptor interaction' and 'IL-17 signaling pathway'.

Based on the transcriptomics results of the present study, future studies should aim to further investigate the specific mechanism underlying the effects of sirolimus in LN and to discover novel drug targets for the treatment of this disease.

\section{Acknowledgements}

Not applicable.

\section{Funding}

The present study was supported by the Clinical Pharmacy Key Specialty Construction Project of Shanghai (grant no. YZ2017/5), The Young Medical Talents of Wuxi (grant no. QNRC020), Young Project of Wuxi Health and Family Planning Research (grant no. Q201706), Wuxi science and technology development guidance plan (medical and health care; grant no. CSZON1744) and the AOSAIKANG pharmaceutical foundation (grant no. A201826).

\section{Availability of data and materials}

The datasets used and/or analyzed during the current study are available from the corresponding author on reasonable request.

\section{Authors' contributions}

ZL made substantial contributions to the conception and design of the present study. DW, XC and MF performed the experiments. DW and XC wrote, reviewed and edited the manuscript. All authors read and approved the final manuscript.

\section{Ethics approval and consent to participate}

The present study was approved by the Animal Care and Use Committee of the Children's Hospital of Fudan University (Shanghai, China), and complied with our institutional regulations.

\section{Patient consent for publication}

Not applicable.

\section{Competing interests}

The authors declare that they have no competing interests.

\section{References}

1. Wang DD, Lu JM, Li Q and Li ZP: Population pharmacokinetics of tacrolimus in paediatric systemic lupus erythematosus based on real-world study. J Clin Pharm Ther 43: 476-483, 2018.

2. Takeuchi T, Tsuzaka K, Abe T, Yoshimoto K, Shiraishi K, Kameda $\mathrm{H}$ and Amano K: $\mathrm{T}$ cell abnormalities in systemic lupus erythematosus. Autoimmunity 38: 339-346, 2005.

3. Rahman A and Isenberg DA: Systemic lupus erythematosus. N Engl J Med 358: 929-939, 2008
4. Gurevitz SL, Snyder JA, Wessel EK, Frey J and Williamson BA: Systemic lupus erythematosus: A review of the disease and treatment options. Consult Pharm 28: 110-121, 2013.

5. D'Cruz DP, Khamashta MA and Hughes GR: Systemic lupus erythematosus. Lancet 369: 587-596, 2007.

6. Liao R, Liu Q, Zheng Z, Fan J, Peng W, Kong Q, He H, Yang S, Chen W, Tang X and Yu X: Tacrolimus protects podocytes from injury in lupus nephritis partly by stabilizing the cytoskeleton and inhibiting podocyte apoptosis. PLoS One 10: e0132724, 2015.

7. Bertsias G, Ioannidis JP, Boletis J, Bombardieri S, Cervera R, Dostal C, Font J, Gilboe IM, Houssiau F, Huizinga T, et al: EULAR recommendations for the management of systemic lupus erythematosus. Report of a task force of the eular standing committee for international clinical studies including therapeutics. Ann Rheum Dis 67: 195-205, 2008.

8. Ji Y, Chen S, Xiang B, Li K, Xu Z, Yao W, Lu G, Liu X, Xia C, Wang Q, et al: Sirolimus for the treatment of progressive kaposiform hemangioendothelioma: A multicenter retrospective study. Int J Cancer 141: 848-855, 2017.

9. Jung KS, Lee J, Park SH, Park JO, Park YS, Lim HY, Kang WK and Kim ST: Pilot study of sirolimus in patients with PIK3CA mutant/amplified refractory solid cancer. Mol Clin Oncol 7: 27-31, 2017.

10. Djebli N, Rousseau A, Hoizey G, Rerolle JP, Toupance O, Le Meur Y and Marquet P: Sirolimus population pharmacokinetic/pharmacogenetic analysis and bayesian modelling in kidney transplant recipients. Clin Pharmacokinet 45: 1135-1148, 2006.

11. Jiao Z, Shi XJ, Li ZD and Zhong MK: Population pharmacokinetics of sirolimus in de novo Chinese adult renal transplant patients. Br J Clin Pharmacol 68: 47-60, 2009.

12. Wataya-Kaneda M, Nakamura A, Tanaka M, Hayashi M, Matsumoto S, Yamamoto K and Katayama I: Efficacy and safety of topical sirolimus therapy for facial angiofibromas in the tuberous sclerosis complex: A randomized clinical trial. JAMA Dermatol 153: 39-48, 2017.

13. Wang DD, Lu JM, Li YZ, Li Q and Li ZP: Population pharmacokinetics of sirolimus in pediatric tuberous sclerosis complex: From real world study. Int J Clin Exp Med 11: 12302-12309, 2018.

14. Zhou L, Du GS, Pan LC, Zheng YG, Liu ZJ, Shi HD, Yang SZ, Shi XJ, Xuan M, Feng LK and Zhu ZD: Sirolimus treatment for cirrhosis or hepatocellular carcinoma patients accompanied by psoriasis after liver transplantation: A single center experience. Oncol Lett 14: 7817-7824, 2017.

15. Zhou L, Pan LC, Zheng YG, Du GS, Fu XQ, Zhu ZD, Song JY, Liu ZJ, Su XZ, Chen W, et al: Novel strategy of sirolimus plus thymalfasin and huaier granule on tumor recurrence of hepatocellular carcinoma beyond the UCSF criteria following liver transplantation: A single center experience. Oncol Lett 16: 4407-4417, 2018.

16. Fu J, Wang Z, Lee K, Wei C, Liu Z, Zhang M, Zhou M, Cai M, Zhang W, Chuang PY, et al: Transcriptomic analysis uncovers novel synergistic mechanisms in combination therapy for lupus nephritis. Kidney Int 93: 416-429, 2018.

17. Yap DYH, Tang C, Chan GCW, Kwan LPY, Ma MKM, Mok MMY and Chan TM: Longterm data on sirolimus treatment in patients with lupus nephritis. J Rheumatol 45: 1663-1670, 2018.

18. Liu J, Yang L, Hou Y, Soteyome T, Zeng B, Su J, Li L, Li B, Chen D, Li Y, et al: Transcriptomics study on staphylococcus aureus biofilm under low concentration of ampicillin. Front Microbiol 9: 2413, 2018.

19. Kim D, Langmead B and Salzberg SL: HISAT: A fast spliced aligner with low memory requirements. Nat Methods 12: 357-360, 2015.

20. Wang K, Li M and Hakonarson H: ANNOVAR: Functional annotation of genetic variants from high-throughput sequencing data. Nucleic Acids Res 38: e164, 2010.

21. Burden CJ, Qureshi SE and Wilson SR: Error estimates for the analysis of differential expression from RNA-seq count data. PeerJ 2: e576, 2014.

22. Kanehisa M and Goto S: KEGG: Kyoto encyclopedia of genes and genomes. Nucleic Acids Res 28: 27-30, 2000.

23. Ashburner M, Ball CA, Blake JA, Botstein D, Butler $\mathrm{H}$ Cherry JM, Davis AP, Dolinski K, Dwight SS, Eppig JT, et al: Gene ontology: Tool for the unification of biology. The gene ontology consortium. Nat Genet 25: 25-29, 2000.

24. Seshan SV and Jennette JC: Renal disease in systemic lupus erythematosus with emphasis on classification of lupus glomerulonephritis: Advances and implications. Arch Pathol Lab Med 133: 233-248, 2009. 
25. Tveita A, Rekvig OP and Zykova SN: Glomerular matrix metalloproteinases and their regulators in the pathogenesis of lupus nephritis. Arthritis Res Ther 10: 229, 2008.

26. Kolset SO, Reinholt FP and Jenssen T: Diabetic nephropathy and extracellular matrix. J Histochem Cytochem 60: 976-986, 2012.

27. Wang D, Zhang G, Chen X, Wei T, Liu C, Chen C, Gong Y and Wei Q: Sitagliptin ameliorates diabetic nephropathy by blocking TGF-beta1/Smad signaling pathway. Int J Mol Med 41: 2784-2792, 2018.

28. Chen X, Wang DD, Wei T,He SM,Zhang GY and Wei QL: Effects of astragalosides from radix astragali on high glucose-induced proliferation and extracellular matrix accumulation in glomerular mesangial cells. Exp Ther Med 11: 2561-2566, 2016.

29. Zhang GY, Wang DD, Cao Z, Wei T, Liu CX and Wei QL: Sitagliptin ameliorates high glucose-induced cell proliferation and expression of the extracellular matrix in glomerular mesangial cells. Exp Ther Med 14: 3862-3867, 2017.
30. Chen X, Yang Y, Liu C, Chen Z and Wang D: Astragaloside IV ameliorates high glucoseinduced renal tubular epithelialmesenchymal transition by blocking mTORC1/p70S6K signaling in HK2 cells. Int J Mol Med 43: 709-716, 2019.

31. Badid C, Desmouliere A, Babici D, Hadj-Aissa A, McGregor B, Lefrancois N, Touraine JL and Laville M: Interstitial expression of alpha-SMA: An early marker of chronic renal allograft dysfunction. Nephrol Dial Transplant 17: 1993-1998, 2002.

32. Cao YH, Lv LL, Zhang X, Hu H, Ding LH, Yin D, Zhang YZ, $\mathrm{Ni} \mathrm{HF}$, Chen PS and Liu BC: Urinary vimentin mRNA as a potential novel biomarker of renal fibrosis. Am J Physiol Renal Physiol 309: F514-F522, 2015.

This work is licensed under a Creative Commons Attribution-NonCommercial-NoDerivatives 4.0 International (CC BY-NC-ND 4.0) License. 